\title{
LIFETIME OPTIMIZATION OF HOT FORGED AEROSPACE COMPONENTS BY LINKING MICROSTRUCTURAL EVOLUTION AND FATIGUE BEHAVIOUR
}

\author{
H. Maderbacher ${ }^{1, \text { a }}$, H.-P. Gänser ${ }^{2, \text { b }}$, M. Riedler ${ }^{3, ~ c}$, M. Stoschka ${ }^{1, d}$, \\ M. Stockinger ${ }^{3, \mathrm{e}}, \mathrm{W}$. Eichlseder ${ }^{1, \mathrm{f}}$ \\ 1) Chair of Mech. Engineering, University of Leoben, Franz-Josef-Str. 18, 8700 Leoben, Austria \\ ${ }^{2)}$ Materials Center Leoben Forschung $\mathrm{GmbH}$, Roseggerstraße 12, 8700 Leoben, Austria \\ 3) Böhler Schmiedetechnik GmbH\&Co KG, Mariazellerstraße 25, 8605 Kapfenberg, Austria \\ ahermann.maderbacher@unileoben.ac.at, bhp.gaenser@mcl.at, \\ cmartin.riedler@bohler-forging.com, ${ }^{\mathrm{c}}$ michael.stoschka@unileoben.ac.at, \\ emartin.stockinger@bohler-forging.com, wilfried.eichlseder@unileoben.ac.at
}

Key words: Optimization, Fatigue, Microstructure, Forging, INCONEL718

\begin{abstract}
Heavy-duty aerospace components are frequently hot forged to satisfy the high requirements concerning their mechanical behaviour. Only the usage of high-performance materials together with a near-optimum manufacturing process enables the production of parts that are at the same time lightweight and mechanically extremely durable. Not only the static properties, but also the fatigue behaviour of Inconel718 is strongly influenced by the material's microstructure resulting from the forging and heat treatment processes. Therefore, the static and fatigue properties may be controlled via the microstructural properties by suitably adjusting the parameters of the manufacturing processes. The present work links the complete forging and heat treatment process to the local distribution of the material's fatigue strength within a component; the effect of the operating temperature is also considered. To this purpose, an empirical model is derived from fatigue tests on specimens with different microstructures at different temperatures. The resulting fatigue strength model is implemented, along with a microstructural evolution model from earlier work [1], into a finite element code in order to predict the local fatigue strength distribution in a component after being subjected to an arbitrary forging process. In a further step, the finite element code is linked to an optimization tool for determining the optimum set of manufacturing process parameters such that the component lifetime is maximized while taking process constraints into consideration.
\end{abstract}

\section{Introduction}

Near-optimum fatigue strength can be reached only if all the influencing parameters as well as their interactions during the whole manufacturing process are known. The most advantageous combination of these parameters can be found in a closed optimization chain which implies the complete manufacturing process. Such a chain could be described as follows. At first, the raw part is subjected to the forging and heat treatment processes. In addition to the mechanical behaviour arising from the forming process, further mechanical influences like residual stresses, the effects of finishing and shot peening, or technological influences like the operation temperature are included. If the local material fatigue strength as well as additional effects such as the influence of mean stress, stress gradient and finishing are understood, it is possible to calculate the component fatigue strength for a specific load spectrum. Finally, an optimization tool can be included at different positions in the simulation chain in order to change the process parameters iteratively such that the highest possible fatigue strength is reached. 


\section{Fatigue Results}

The finite element (FE) software package Deform ${ }^{\mathrm{TM}}$ is used for numerical simulation of the forging and heat treatment process. In earlier work [1], a microstructural approach was implemented in this software. As a result, many microstructural parameters can be predicted by the forging and heat treatment simulation. In a further step, fatigue tests were conducted on specimens having undergone different forging processes [2]. Numerous micrographs from these specimens were analyzed in terms of more than twenty microstructural parameters describing grain size, shape and orientation. In a subsequent step these parameters were eliminated which were either linearly dependent on another parameter, or showed no correlation to the fatigue strength. Despite the significant number of parameters available, the smallest scatter between experimentally determined and predicted fatigue strength was reached if only the grain size was considered according to the following equations:

$$
\begin{aligned}
& S=6.55 \cdot \exp \left(-1.56 \cdot \frac{E C D}{5715}\right)+5.87 \cdot \exp \left(-0.025 \cdot \frac{E C D}{5715}\right) \\
& \tau_{\text {Temp }}=-1.729 \cdot 10^{-11} \cdot T^{4}+2.81 \cdot 10^{-8} \cdot T^{3}-5.397 \cdot 10^{-6} \cdot T^{2}+8.622 \cdot 10^{-4} \cdot T+1.504 \\
& S_{\text {cal }}=S \cdot \tau_{\text {Temp }}
\end{aligned}
$$

Here, $S$ denotes the standardized fatigue strength parameter and $E C D$ is the equivalent circle diameter determined from the micrographs. $S_{\text {cal }}$ is the fatigue strength parameter corrected by $\tau_{T e m p}$ for the influence of the operating temperature $T$. This relation between grain size and fatigue strength was implemented into Deform ${ }^{\mathrm{TM}}$, thereby allowing the determination of the local fatigue strength in each node of the finite element model.

Table 1: Parameters which can be handled in the course of optimization

\begin{tabular}{ll}
\hline variables & manipulation capabilities (initial conditions) \\
\hline \multirow{2}{*}{ node temperature } & initial billet temperature \\
& die temperatures \\
environment temperature during forging process & furnace temperature during heat treatment \\
environment temperature & friction between dies and work piece \\
friction coefficient & heat transfer between dies and work piece \\
heat transfer & die speed \\
movement control & resting time \\
& reheating time \\
number of steps & transfer time \\
\hline
\end{tabular}




\section{Sensitivity analysis}

As a first example for the system response a turbine disk is investigated. The forging process consists of twenty single steps, which are shown in Table 2, including the valid parameter ranges. As single scalar objective function $O b j F n c$, wich validates the quality of the material fatigue strength in the component, the inverse mean value of the fatigue strength multiplied by a constant factor was chosen, in order to get values greater or equal to 1 ,

$$
O b j F n c=\frac{\left(\sum E l\right) * 10^{3}}{\sum S_{c a l}} .
$$

Here, $\Sigma E l$ is the number of elements in the FE model and $\mathrm{S}_{\mathrm{cal}}$ is the local fatigue strength computed according to Equation (3).

For a better understanding of the process, a sensitivity analysis was conducted where all the parameters were varied within the defined limits. In addition to the performance of the overall process also the near optimum parameter combination and the sensitivity of the objective function with respect to each manipulation parameter is documented. The smallest and thus best value of the objective function was found, from the sensitivity analysis, to be 1.17 .

Figure 1 shows on the one hand the system response of the sensitivity analysis and on the other hand the sensitivities of the initial conditions of the individual manipulation parameters. The sensitivities of each parameter show the rough system response if only one parameter is changed. However, the interactions between the different initial conditions of the parameters are neglected. Overall, it is seen that a value near the lower boundary of the billet temperature, low die speed and a long time gap between the forging steps have positive effects on the fatigue behaviour. The reheating temperature in the furnace has no influence within the temperature range chosen, because the solvus temperature of the delta-phase is never reached.

\section{Optimization}

For optimizing the forging process, the open source Software Dakota ${ }^{\mathrm{TM}}$ is used which offers numerous different optimization strategies. An interface between the FE software and the optimization software was created which works in simple terms as follows. At the beginning the lower and upper limits of the optimization parameters are defined. In the following step, an FE input file is generated where the parameter values of the current optimization run are stored. From the FE results of the forging or heat treatment process, the objective function (eq. 4) is generated during post-processing; this objective function has to be minimized in order to maximize the fatigue strength. The optimization tool compares the current value of the objective function to the results from previous runs and estimates then a new set of parameter values for the next simulation run. Table 1 summarizes the parameters which can be directly manipulated with this interface in the Deform $^{\mathrm{TM}}$ simulation. Some examples for conditions which are manageable in this way are also mentioned in this table.

The challenge of the present application is to find an appropriate optimization strategy. The demands on the optimizer are stability, fast convergence as well as the capability to find not only a local optimum, but also the global one.

Different optimization strategies were tested. These strategies contain both gradient and gradientfree methods (Fig. 2). In general, the choice of the optimization strategy and optimization methods depends on the number of changeable parameters [3], the smoothness of the objective function, the numerical noise, and the computational effort per optimization iteration. The gradient-based strategies promise quadratic convergence in the vicinity of a local optimum but cannot ensure to find the global optimum. Also, these methods are sensitive to numerical noise and discontinuities of the objective function, and their performance deteriorates with increasing number of parameters. Gradient-free methods are able to handle a higher number of parameters and show better performance in presence of discontinuities and numerical noise. As a disadvantage, the low rate of convergence rate near the optimum must be mentioned. 
As typical deterministic gradient-free algorithms, the global direct search method and the pattern search method were considered [4]. From the stochastic methods, an evolutionary algorithm and a genetic algorithm were applied.

None of the gradient-based methods produced satisfying results, which could have been caused by the big number of parameters on the one hand, or by the choice of the starting values on the other hand. The deterministic gradient-free methods do not show acceptable convergence after 500 iterations either. This effect could be caused by the fact that the pattern search method is also based on the idea of local search, and therefore rather sensitive to the starting point. The global direct search method needs too many iterations for convergence.

Both the evolutionary algorithm and the genetic algorithm (as a special case of an evolutionary algorithm) show fast convergence in the beginning of the optimization process; unfortunately, the convergence rate decreases markedly as the solution approaches its optimum.

Table 2: Forging process of reference component

\begin{tabular}{|c|c|c|c|c|c|}
\hline step & operation & deviations from bas & process & & \\
\hline 1 & initial billet temperature in furnace & billet temperature & $-2 \%$ & - & $+3 \%$ \\
\hline 2 & transfer from furnace to screw press & & & & \\
\hline 3 & resting on lower die & & & & \\
\hline 4 & pre forming in screw press & die speed & $-17 \%$ & - & $+67 \%$ \\
\hline 5 & resting on lower die & & & & \\
\hline 6 & free resting & & & & \\
\hline 7 & resting on lower die & & & & \\
\hline 8 & pre-forming in screw press & die speed & $-17 \%$ & - & $+67 \%$ \\
\hline 9 & resting on lower die & & & & \\
\hline 10 & transfer from screw press to furnace & & & & \\
\hline 11 & reheating in furnace & furnace temperature & $-2 \%$ & - & $+3 \%$ \\
\hline 12 & transfer from furnace to screw press & & & & \\
\hline 13 & resting on lower die & & & & \\
\hline 14 & final pressing in screw press & die speed & $-17 \%$ & - & $+67 \%$ \\
\hline 15 & resting on lower die & & & & \\
\hline 16 & free resting & resting time & $-0 \%$ & - & $+100 \%$ \\
\hline 17 & resting on lower die & & & & \\
\hline 18 & final pressing in screw press & die speed & $-17 \%$ & - & $+67 \%$ \\
\hline 19 & resting on lower die & & & & \\
\hline 20 & air cooling & & & & \\
\hline
\end{tabular}




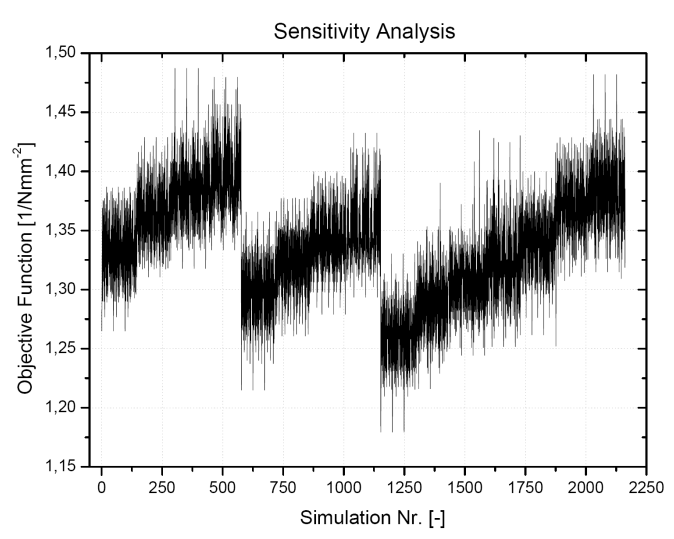

Fig. 1- a: Unsorted results for sensitivity analysis

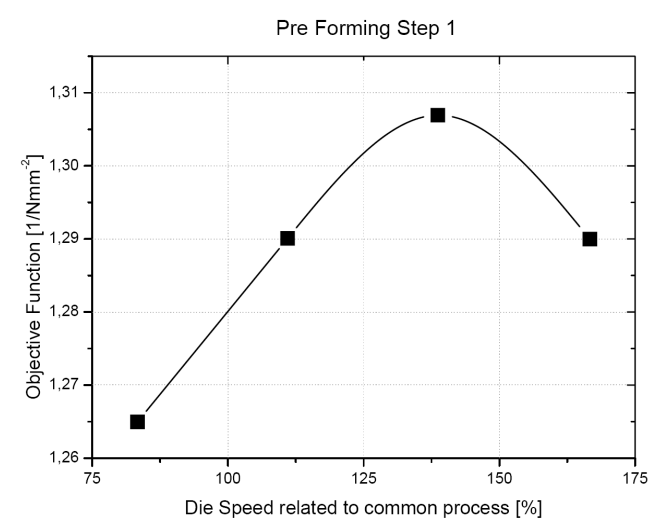

Fig. 1- c: Sensitivity to the die speed in the first pre-forming step

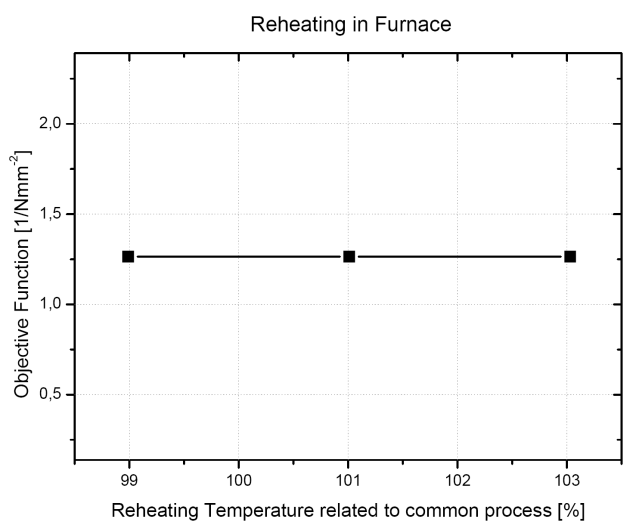

Fig. 1- e: Sensitivity to the furnace temperature

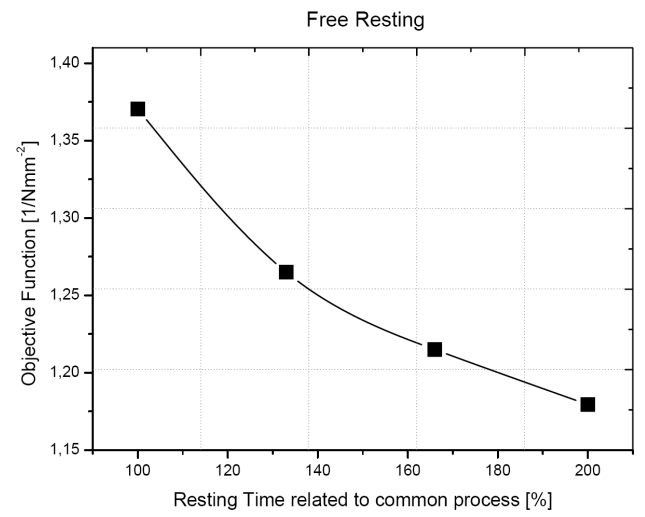

Fig. 1- g: Sensitivity to the duration of free resting

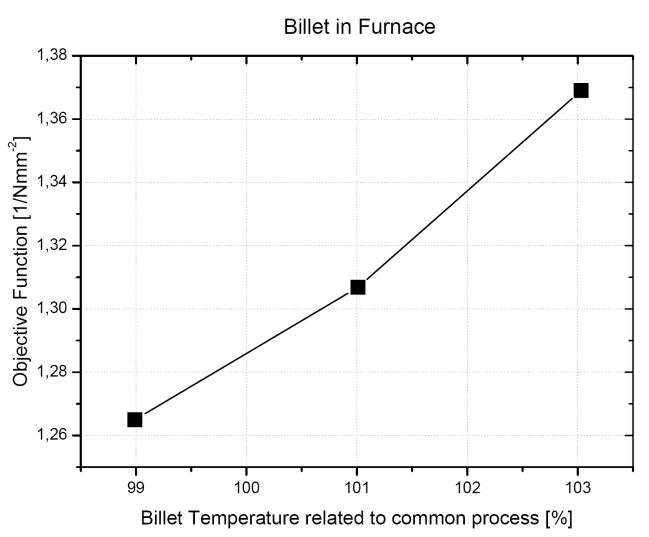

Fig. 1- b: Sensitivity to the initial billet temperature

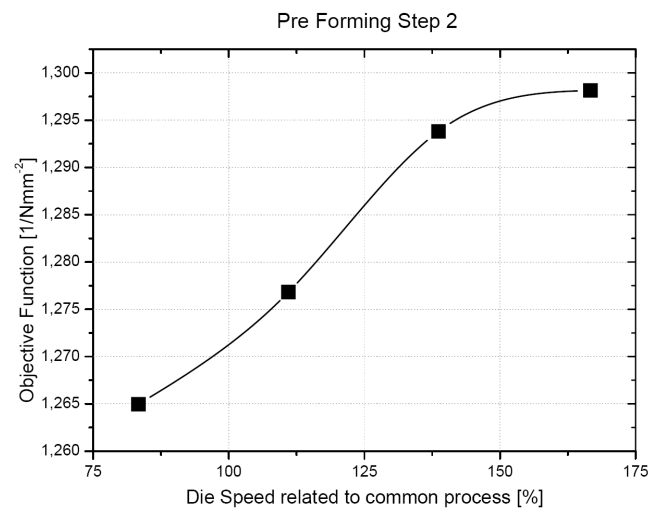

Fig. 1- d: Sensitivity to the die speed in the second pre-forming step

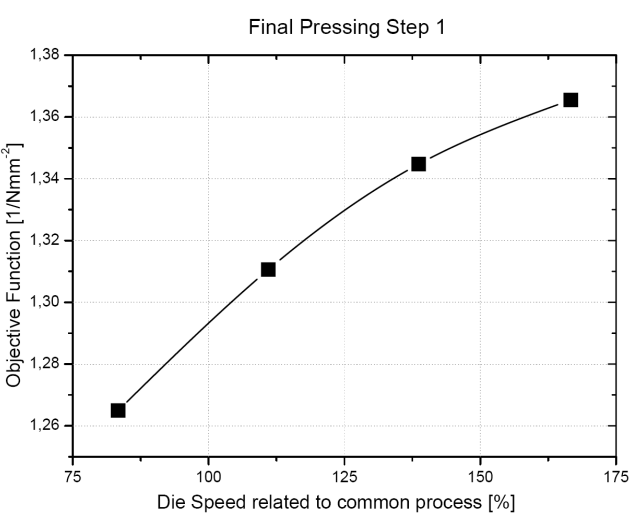

Fig. 1- f: Sensitivity to the die speed in the first final pressing step

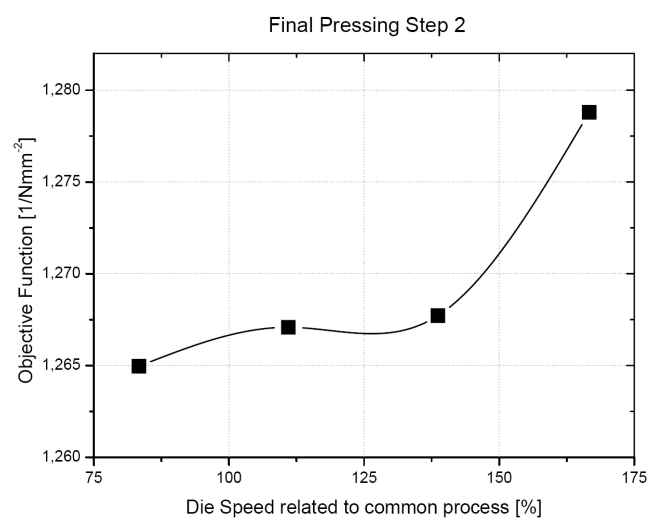

Fig. 1- h: Sensitivity to the die speed in the second final pressing step 


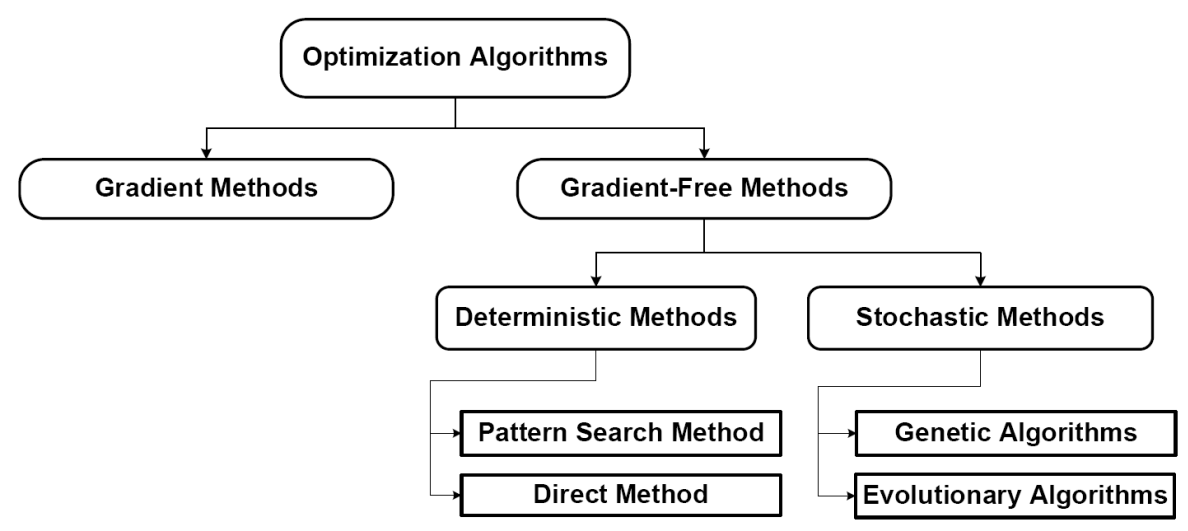

Fig. 2: Different Optimization strategies tested

\section{Conclusion}

A fatigue model based on the microstructure has been implemented into a finite element code in order to predict the local fatigue strength distribution in a component after being subjected to an arbitrary forging process. This finite element code is linked to an optimization tool for automatically determining the optimum set of forging process parameters such that the component lifetime is maximized. Several optimization strategies have been compared with respect to computational effort and rate of convergence. Therefore a Hybrid-Optimization strategy has to be chosen which combines the advantageous properties of the global stochastic methods with the local gradient based or deterministic methods. In this way the vicinity of the optimum can be quickly reached by the global search method before the local method finds the parameter set for the absolute optimum.

\section{Acknowledgements}

Financial support by the Austrian Federal Government and the Styrian Provincial Government, represented by Österreichische Forschungsförderungsgesellschaft $\mathrm{mbH}$ and Steirische Wirtschaftsförderungsgesellschaft $\mathrm{mbH}$, within the research activities of the K2 Competence Centre on "Integrated Research in Materials, Processing and Product Engineering", operated by the Materials Center Leoben Forschung GmbH under the frame of the Austrian COMET Competence Centre Programme, is gratefully acknowledged.

\section{References}

[1] Stockinger, M., Mikrostrukturelle Simulation des Gesenkschmiedens von Nickelbasis Legierungen (Microstructural Simulation of Die Forging of Nickel Base Alloys; in German), Ph.D. Thesis, Technical University of Graz 2003

[2] Stoschka, M., Assessment of lifetime calculation of forged IN718 aerospace components based on a multi-parametric microstructural evaluation, in: Proc. Superalloy 2008

[3] Meywerk, M., CAE-Methoden in der Fahrzeugtechnik, Springer Berlin Heidelberg, 2007

[4] Eldred, M. S., DAKOTA Version 4.2 User's Manual, Sandia National Laboratories, October 2006 